\title{
Server Virtualization in Data Center of Higher Vocational College
}

\author{
Cong Cheng ${ }^{1, a}$, Aiqing Chen ${ }^{2, b}$ \\ ${ }^{1}$ Information Center,Beijing Polytechnic College,Beijing,100042,China \\ ${ }^{2}$ Dept. of Mathematics, Jiaozuo Teachers College,Jiaozuo,454000,China \\ âE-mail:Cong_cheng@sina.com, ${ }^{\mathrm{b}}$ E-mail:niuxt@163.com
}

Keywords: Virtual server; Server virtualization;Data center

\begin{abstract}
This paper proposes an implementation plan of VMware-vSphere-based server virtualization that targets at the irrational utilization of hardware resources in higher vocational colleges, management and maintenance difficulties, poor compatibility of software \& hardware, and other concerns. By building a dynamic pool of virtualized resources, it manages to improve the resource utilization, achieve the integration of business systems, and enhance their security \& availability.
\end{abstract}

\section{Introduction}

Vocational colleges have constantly kicked off the overall planning and construction of digital campus in recent years, with prominent issues including independent working of software \& hardware in functional departments that results in redundant construction; lack of safety precautions, low equipment utilization, slowdown in operation \& maintenance ability, and waste of IT resources. Server virtualization has been the answer to the above problems.

\section{Concerns of data center of higher vocational colleges}

With the acceleration of digital campus construction in higher vocational colleges, drawbacks of traditional physical data center have exposed gradually. The server and system distribution (Table 1) of a vocational college in Beijing reveals lots of concerns in management and resource sharing for IT Dept.

Management and maintenance are difficult.As applications for personnel, finance, education, portals and web-based teaching are distributed on more than 40 servers that work independently, and failure of one server will lead to the breakdown of corresponding system. Frequent single point of failure (SPOF) has brought mass management and maintenance as well as difficulty in fault warning and unified management. In addition, the poor scalability of "island-like" data center makes new network, server and storage infrastructure a necessity for new business.

Hardware resources cannot be utilized rationally.Dozens of business systems in terms of, for instance, OA, mail, personnel management and research management, have different functions, service objects and frequency of utilization. Take OA server for example. It runs at full capacity during work hours but is idle after work; educational system is seldom used at other times but visited frequently at the end of a semester for course choosing, examination affairs and results uploading, causing insufficient system concurrency and even interruption of service response. The independence of servers makes it difficult to share calculation, storage and network resources, to optimize the system performance, and to utilize the hardware resources rationally.

Software \& hardware compatibility is poor.Functional departments purchase (or develop) hardware equipment and diverse business systems based on their needs at different time. Servers in the data center may contain more than a dozen models of IT companies, types and versions of operating systems (OS), as well as application of about ten databases. It is very likely that OS and application software cannot function on a new hardware platform when a system is migrated to a new server, and old codes sometimes cannot be migrated successfully, thus worsening the incompatibility between hardware and software. 
Construction and operation costs of the center apparatus room (CAR) are high.As it has strict environmental requirements, there are thus clear specifications on temperature, humidity, cooling, static electricity, electromagnetic interference, lightning protection, monitoring and management. A hundred servers in some vocational colleges require huge investment for daily operation, together with large power consumption of air-conditioning \& servers and the growing demands for room space. To minimize the number of physical servers and to reduce energy consumption has aroused general concern.

Therefore, data center in vocational colleges should put an emphasis on decreasing the operating costs, reducing the management complexity, allocating hardware and software resources rationally, improving the system compatibility and enhancing data security and stability.

\section{Necessity of server virtualization}

More and more vocational colleges prefer to deal with the increasing pressure of data center via virtualization, which is a broad term referring to that computing elements run on a virtual rather than a real basis, so as to expand the capacity of hardware and simplify the reconfiguration of software, divided into server virtualization, network virtualization and storage virtualization.

More specifically, server virtualization means to allocate resources from a logical perspective, in which a server is used to simulate multi-server in parallel, allowing multiple OS to run on one platform and applications to work independently from each other in separate space. Virtualization enables a server to act as multiple virtual servers, each with its own physical resources such as CPU and storage, thus separating software from hardware, and users do not need to consider the background hardware when operating systems and software. Currently, the mature virtualization solution for data center generally accepted by colleges is VMware Infrastructure from VMware, Inc.

Server virtualization is advantageous in that it helps with the virtual partition of physical server dynamically based on the real-time business needs, and enables each virtual machine to be made full use of as a physical server, thus greatly improving the utilization of each server, storage, and other resources. Secondly, IP-address-based remote management function enables the manager to monitor and manage the virtual server at BIOS-level anywhere via the Internet, along with remote start, centralized management, remote control of CPU, memory and running conditions of the host and virtual machine, changes in the hardware configuration, and installation \&commissioning of various software on the virtual machine, so as to reduce the management work. In case of server failure or maintenance and upgrades, virtualization helps to migrate the tasks on the virtual server dynamically and lively to a physical server; even if the server halts, the software applications on it may work smoothly. Upon the server is repaired, the system will move the tasks back to achieve load balancing and ongoing functioning of all applications, ensuring the delivery of quality service. In addition, by integrating various businesses on one physical server, virtualization has decreased the number of physical servers and solved the problem of scattered and complex infrastructure. Just think what if a CAR reduces the number of its servers from 80 to 10, the environment and lines will be clean and neatly, and the power consumption will be reduced greatly.

\section{Implementation plan}

Take the data center of a vocational college as an example, whose current servers and OS are partly shown (Table 1): 
Table 1.Servers and OS distribution of a vocational college

\begin{tabular}{|l|l|l|l|}
\hline \multicolumn{1}{|c|}{ Brand } & \multicolumn{1}{|c|}{ Model } & \multicolumn{1}{c|}{ OS } & \multicolumn{1}{c|}{ Service } \\
\hline IBM & X3650 & Win2003 sp2 & Teaching resource platform \\
\hline Fujitsu & $\begin{array}{l}\text { Fujitsu } \\
\text { M3000 }\end{array}$ & Solaris & Data acquisition platform \\
\hline IBM & X3650 M3 & Win2008 sp1 & BlackBoard \\
\hline IBM & X3650 M3 & Win2008 sp1 & BlackBoard \\
\hline IBM & X 3650 M2 & RedHat & Shared professional teaching resource platform \\
\hline IBM & X 346 & Win2000 sp4 & Scientific research system management \\
\hline IBM & X 3650 & Win2003 sp2 & Web-based teaching evaluation system \\
\hline HP & DL580 G5 & Win2008 & Educational administration system \\
\hline \multirow{2}{*}{ IBM } & X 3550 & Win2003 sp2 & Financial system \\
\cline { 2 - 5 } & Linux & Financial system \\
\hline IBM & X 345 & Win2003 sp2 & Work organization system \\
\hline IBM & X 345 & Win2003 sp2 & web \\
\hline IBM & X 345 & Win2000 sp4 & Old home page \\
\hline IBM & X 3650 & Win2008 sp1 & Data service for movie network \\
\hline IBM & X3650 & Linux & E-mail filtering system \\
\hline IBM & X 3650 & Linux & Mailing system \\
\hline IBM & X 345 & FreeBSD & DNS \\
\hline IBM & X 3650 & Win2003 sp2 & $\begin{array}{l}\text { Monitoring system for CAR temperature and } \\
\text { humidity }\end{array}$ \\
\hline IBM & X 345 & Win2003 sp2 & FTP \\
\hline IBM & X 346 & Linux & Integrated network management system \\
\hline$\ldots$ & $\ldots$ & $\ldots$ & $\ldots$ \\
\hline
\end{tabular}

The college has more than 60 servers for different business systems. When developing the server virtualization plan, the IT Dept., through full discussions, learned that not all services in the data center could be virtualized, such as database system and video-on-demand service, which require lots of disk I/O and memory during their operation. Services suitable for virtualized servers, include webbased teaching platform, scientific research system, personnel management system, courseware service, mailing service, network management system, educational administration system, financial system, and teaching resource platform, etc.

To save money, the plan takes 7 existing high-end IBM 3650 servers as physical servers (each with 2-way dual-core CPU, $128 \mathrm{G}$ memory and x86 server); since high configuration is unnecessary for vCenter Server, any IBM 345 server will be a powerful centralized virtualization control tool used for the management of 7 ESX Servers as well as the unified control and management of the entire data center. At a proportion of approx. 5:1, more than 30 virtual servers have been provided in the plan, onto which business systems will be migrated.

In terms of software, 14 sets of Academic VMware vSphere 5 Enterprise and 1 set of Academic VMware vCenter Server 5 Standard for vSphere 5 ( Per Instance) will be required for all CPUs, along with three years of software services in general. Plan-based topology diagram (Fig. 1)

\section{Conclusion}

In view of the actual situation of data center of a vocational college, VMware vSphere is used for 
server virtualization in order to save money, facilitate the management and improve the security. Such virtual server, in terms of features, performance and operations, is equivalent to the traditional single physical server, and can be installed with Windows \& Linux OS, followed by various applications. This helps to form a dynamic pool of virtualized resources, improve the utilization of resources effectively, integrate business systems perfectly, enhance the system security \& availability and facilitate the management, thus achieving the perfect integration of server virtualization.

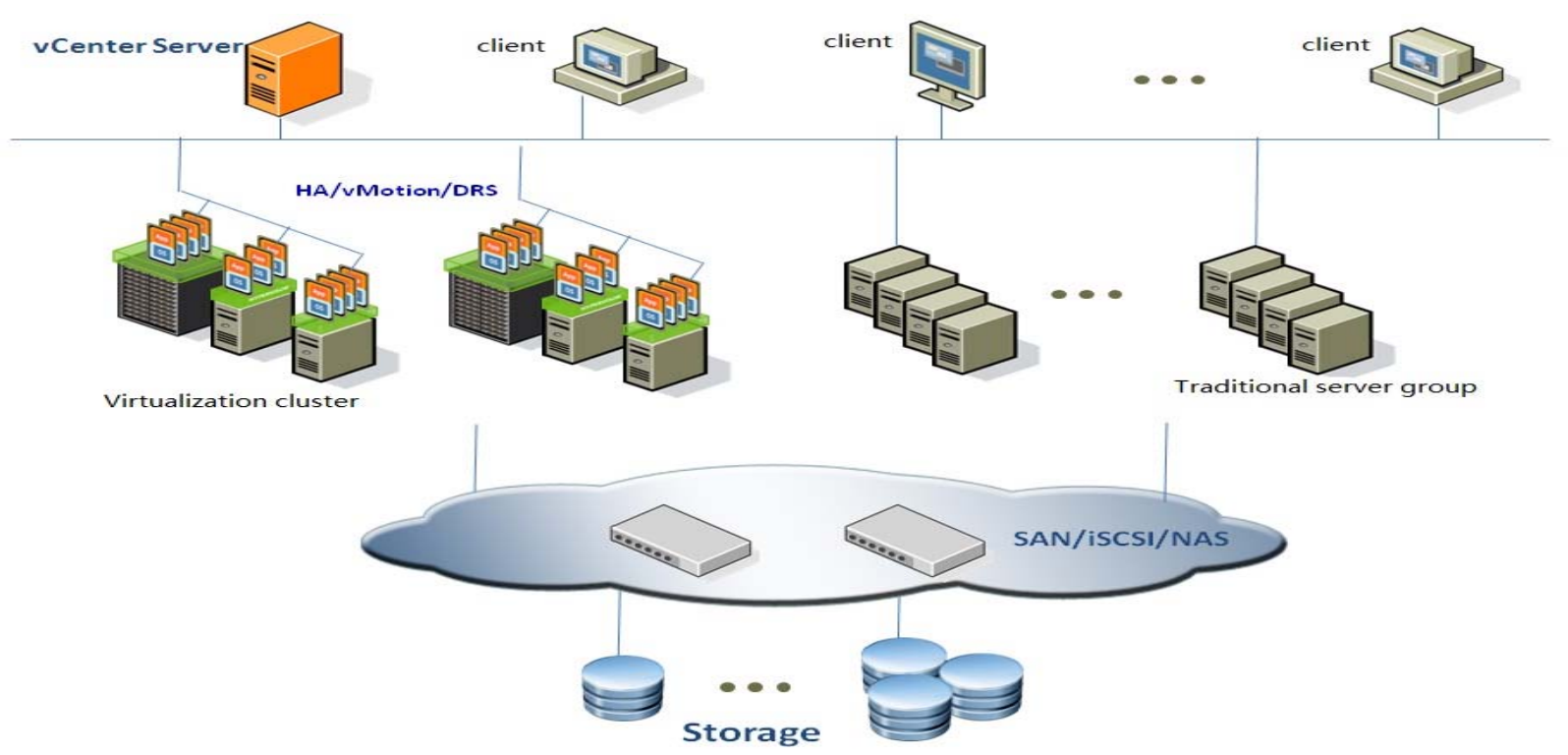

Figure 1. Topology diagram of a vocational college based on server virtualization plan

\section{References}

[1] Laifu Wang \& Jun Sheng, et al., Research on the Security of iCloud Application [J], Telecom Science, 2010 (6): pp. 67-69.

[2] Zhengwu Yuan \& Qi Li, Current Situation \& Trend of iCloud Application [J], Digital Communications, 2010 (6): pp. 37-40

[3] Gang Shi, Construction \& Application of Shared Servers of Wuhan University Based on IaaS [N], China Education Network, 2010 (10): pp. 36-37.

[4] Qing Shi, Research on Virtualization Construction of College Data Center Based on VMware vSphere [D], 2010.7.

[5] Li Ruixing, Study of Teaching Applications in Higher Polytechnic Colleges Based on Case Teaching Method [J], Study of Higher Education, 2010（8） : 173-174 